\begin{tabular}{|c|c|c|}
\hline $\begin{array}{c}\text { ISSN 2525-4812 (versão online) } \\
\text { ISSN 2238-7641 (versão impressa) } \\
\text { http://www.revistaterceiramargem.com/ } \\
\text { index.php/terceiramargem/index }\end{array}$ & $\begin{array}{c}\text { Recebido em: 19/6/2019 } \\
\text { Aprovado em: 30/2/2020 } \\
\text { Período de publicação: jan./jun., } 2020\end{array}$ & $\begin{array}{c}\text { Revista Terceira } \\
\text { Margem Amazônia } \\
(\text { v. } 6 \cdot \mathrm{n} .14 \cdot \text { Jan./Jun. } 2020)\end{array}$ \\
\hline
\end{tabular}

Como citar o artigo:

BATISTA, S. C. P.; COSTA, S. C. F. das C.; COSTA, F. S. da; DIAS JÚNIOR, L. As dificuldades dos agricultores familiares na produção orgânica na feira agroufam de Manaus, AM. Revista Terceira Margem Amazônia, v. 6, n. 14, p. 09-15, 2020. DOI: http://dx.doi.org/10.36882/2525-4812.2020v6i14p9-15

\title{
AS DIFICULDADES DOS AGRICULTORES FAMILIARES NA PRODUÇÃO ORGÂNICA NA FEIRA AGROUFAM DE MANAUS, AM
}

\author{
Samara Claudia Picanço Batista ${ }^{1}$ \\ Sarah Caroline Ferreira das Chagas Costa ${ }^{2}$ \\ Francimara Souza da Costa \\ Luiz Dias Júnior ${ }^{4}$
}

\begin{abstract}
Resumo: Existe uma crescente demanda da população em geral por alimentos mais saudáveis e que causem menos impacto ambiental, porém a agricultura familiar pouco opta pelo método orgânico de produção. Nesse contexto objetivou-se, neste artigo, entender o cenário local apresentando as motivações e dificuldades dos produtores de orgânicos situados na feira Agroufam. Para avaliar a realidade desses agricultores foram feitos levantamentos documentais, estudo de caso e aplicação de questionários. Os resultados apontaram que a falta de orientação técnica especializada e o alto custo da certificação são as principais dificuldades; no entanto, a maior motivação é o fornecimento de alimentos saudáveis à população, cuja produção não agride o meio ambiente.
\end{abstract}

Palavras-chave: agricultura orgânica, alimento saudável, desafios, perspectivas.

\section{THE DIFFICULTIES OF FAMILY FARMERS IN ORGANIC PRODUCTION AT THE AGROUFAM FAIR OF MANAUS, BRAZIL}

\begin{abstract}
There is a growing demand from the general population for healthier foods that cause less environmental impact, but family farming has little choice for the organic production method. There are countless causes that discourage the small producer and the lack of resources and technical assistance are the main obstacles. In this context, this article aimed to understand the local scenario showing the motivations and difficulties of organic producers located in the Agroufam fair. In order to evaluate the reality of these farmers, documentary surveys, case studies and questionnaires were carried out. The results pointed out that the lack of specialized technical guidance and the high cost of certification are the main difficulties and the greatest motivation is the provision of healthy food to the population whose production does not harm the environment.
\end{abstract}

Key words: organic agriculture, healthy food, challenges, prospects.

\footnotetext{
${ }^{1}$ Engenheira de alimentos, Manaus, AM. E-mail: samara_claudia18@outlook.com

${ }^{2}$ Engenheira de alimentos, Manaus, AM. E-mail: sarah23caroline@gmail.com

${ }^{3}$ Engenheira-agrônoma, D. Sc.em Desenvolvimento Sustentável do Trópico Úmido, professora da Universidade Federal do Amazonas, Manaus, AM. E-mail: francimaracosta@yahoo.com

${ }^{4}$ Engenheiro-agrônomo, Manaus, AM. E-mail: diasjunior.luiz@gmail.com
} 


\section{Introdução}

A agricultura orgânica é caracterizada pela restauração da fertilidade do solo por meio de processos biológicos, procurando promover a saúde dos seres humanos e o equilíbrio ambiental sem a utilização de agroquímicos (BARROS; SILVA, 2010). A produção orgânica pode ser um caminho a ser percorrido em busca da sobrevivência harmônica do ser humano com o seu planeta, uma vez que o desenvolvimento sustentável mostra que as necessidades da presente geração devem ser atendidas sem sacrificar a possibilidade de as gerações futuras também atenderem suas próprias necessidades (MAZZOLENI; NOGUEIRA, 2006).

Penteado (2000) afirma que a agricultura orgânica tem por princípio estabelecer sistemas de produção com base em tecnologias de processos, ou seja, um conjunto de procedimentos que envolvam a planta, o solo e as condições climáticas, produzindo um alimento sadio e com suas características e sabor originais que atendam às expectativas do consumidor.

De acordo com França et al. (2010), a agricultura familiar está localizada em ambiente hostil, onde são impostas características de competitividade, seleção e consequentemente exclusão, pertencentes ao sistema capitalista. Porém, a cooperação se faz importante no momento de superar as dificuldades e para que, de forma autônoma, possa conduzir a ação coletiva dos agricultores familiares na construção de identidades locais, fortalecendo assim a cultura além de preservar a diversidade regional.

A agricultura orgânica apresenta desafios, como: custos durante o processo de conversão do sistema convencional para o orgânico e custos de certificação; falta de assistência técnica da rede pública; dificuldades de acesso ao crédito bancário e investimentos em tecnologia; e escassez de pesquisa científica em agricultura orgânica (BARBOSA; SOUSA, 2012).

Este artigo teve como principal objetivo determinar as mais importantes dificuldades na produção orgânica da feira e ressaltar quais são os motivos que levaram os agricultores, objeto desta pesquisa, a inserir o modo de produção agroecológica.

\section{Metodologia}

A feira é uma iniciativa do Núcleo de Socioeconomia da Universidade Federal do Amazonas (Nusec - Ufam), em funcionamento desde o ano 2002, com atividade todos os meses nas primeiras quintas-feiras e sextas-feiras, nos blocos 1 e 2 da Faculdade de Ciências Agrárias (FCA), setor sul do campus universitário, situado na Avenida General Rodrigo Otávio, Bairro Coroado I.

A metodologia de exploração dos dados do estudo utilizou como base metodológica a pesquisa descritiva, pois pretendeu descrever as características de determinada população ou fenômeno, ou estabelecer relação entre variáveis cujas características são conhecidas (FREITAS et al., 2015). A partir dessa base foram utilizados os procedimentos de estudo de caso, pesquisa bibliográfica e pesquisa documental como ferramentas para levantamento de dados, trabalho de campo com coleta de dados por meio de entrevistas estruturadas, e o problema foi estudado sob a ótica qualitativa. 
As entrevistas foram realizadas aplicando-se questionário previamente estruturado e com questões semiabertas. Foram gravados e anotados comentários e observações dos agricultores; desta forma, a pesquisa teve também caráter de entrevista aberta. Coletaram-se informações sobre os municípios relacionadas à produção, às dificuldades enfrentadas, à motivação no modo de produção orgânica, bem como entrevistas de caráter aberto, dirigidas aos produtores da Agroufam. Como assinalado, foram coletados dados em seis barracas de produtores da feira.

O objetivo dessa fase foi estabelecer contato e obter informações necessárias para a elaboração do artigo. A pesquisa em fontes secundárias consistiu de leituras de artigos científicos. Para resguardar a identidade dos entrevistados, estes foram relatados neste estudo como produtores: A, B. C, D, E e F. As entrevistas foram conduzidas in loco, no horário de funcionamento da feira.

\section{Percepções do Produtor de Orgânicos da Feira Agroufam: Dificuldades e Mo- tivações}

Observa-se, na Tabela 1, as principais dificuldades enfrentadas pelos agricultores familiares, o período de ingresso na produção orgânica e as motivações dos produtores da feira da Agroufam.

Tabela 1. Dificuldades e motivações dos produtores orgânicos da feira da Agroufam.

\begin{tabular}{|c|c|c|c|}
\hline $\begin{array}{l}\text { Município de } \\
\text { produção }\end{array}$ & $\begin{array}{l}\text { Período de ingresso na } \\
\text { produção orgânica }\end{array}$ & Dificuldades enfrentadas & $\begin{array}{l}\text { Motivação no modo de } \\
\text { produção orgânico }\end{array}$ \\
\hline Careiro da Várzea & + de 3 anos & $\begin{array}{l}\text { Transporte e locomoção, } \\
\text { perda de produção }\end{array}$ & $\begin{array}{l}\text { Devido à fidelidade dos } \\
\text { consumidores }\end{array}$ \\
\hline Careiro da Várzea & 8 anos & $\begin{array}{l}\text { Manter produção orgâni- } \\
\text { ca devido ao custo mais } \\
\text { elevado }\end{array}$ & $\begin{array}{l}\text { A grande quantidade de } \\
\text { agrotóxicos que é ingerida } \\
\text { por ano }\end{array}$ \\
\hline Presidente Figueiredo & 2 anos & Transporte e locomoção & $\begin{array}{l}\text { Devido à grande procura por } \\
\text { produtos de origem agroe- } \\
\text { cológica }\end{array}$ \\
\hline Rio Preto da Eva & 3 anos & $\begin{array}{l}\text { Perda do produto, falta de } \\
\text { auxílio técnico, certificação }\end{array}$ & $\begin{array}{l}\text { Devido à produção orgânica } \\
\text { ser mais saudável }\end{array}$ \\
\hline Careiro da Várzea & 6 anos & $\begin{array}{l}\text { Perda de produção, transpor- } \\
\text { te, auxílio técnico, controle } \\
\text { de pragas }\end{array}$ & $\begin{array}{l}\text { Devido às doenças que } \\
\text { podem causar em razão de } \\
\text { alimentação inadequada }\end{array}$ \\
\hline
\end{tabular}

Dentre as dificuldades apontadas pelos produtores foram destacadas as seguintes: transporte e locomoção, perda de produção, custo mais elevado no modo de produção, auxílio técnico, certificação e controle de pragas.

Os agricultores entrevistados são de outros municípios e se encontram fisicamente mais distantes da cidade de Manaus. Segundo Finatto e Corrêa (2010), o deslocamento a grandes distâncias, além de exigir maiores gastos econômicos, pode resultar em perda de alguns produtos, principalmente as hortaliças, exigentes em cuidados no transporte. 
O agricultor indicou como dificuldade o maior custo na produção orgânica, uma vez que os insumos têm custo mais elevado. Santos e Monteiro (2004) também constataram maiores custos de produção na agricultura orgânica em razão de maior demanda de mão de obra e alguns insumos, bem como da produção em baixa escala.

Entretanto, o baixo nível de conhecimento das práticas a serem adotadas também se torna um fator que contribui para aumentar os custos de produção, pois o manejo inadequado na produção orgânica pode resultar em perdas aos agricultores. Freitas (2002) explica que a agricultura orgânica pode reduzir custos e ser tão rentável quanto o sistema convencional ou superior. Porém, é essencial que os agricultores invistam na compreensão das práticas e processos preconizados pela agricultura orgânica para otimizá-los e obter melhores resultados.

A falta de auxílio técnico foi uma das dificuldades mais citadas pelos agricultores. Uma das agricultoras afirmou: "Tenho até $60 \%$ de perda do tomate tipo cereja, um dos mais procurados pelos clientes". Para que a agricultura familiar possa produzir maior quantidade de alimentos e com melhor qualidade, torna-se indispensável a atuação proativa da assistência técnica nesse processo. Desse modo, serviços de assistência técnica e extensão rural, quando bem estruturados e fortes, são capazes de transferir as tecnologias geradas pela pesquisa aos agricultores, possibilitando o emprego de novas dinâmicas nas formas de produção, contribuindo para a promoção do desenvolvimento rural (LOMBA et al., 2013).

Há necessidade de maior informação entre os agricultores acerca das técnicas a serem adotadas, especialmente quanto ao controle eficiente de pragas e doenças. No entanto, também se admite a falta de pesquisas mediante o amplo conjunto de práticas e processos relativamente complexos quando se trata dos mais diversos agroecossistemas brasileiros. Diante dessa realidade, os agricultores acabam sendo os agentes mais onerados nesse processo ao saberem que, em muitos casos, sem acesso à informação, buscam gerenciar a produção a partir de tentativas empíricas que acabam resultando em erros e acertos (CAMPANHOLA; VALARINI, 2001; GOMES et al., 2017).

A burocracia para obter a certificação foi enfatizada por alguns agricultores, entretanto alguns não tiveram problema para a obtenção do documento. Conforme Campanhola e Valarini (2001), a certificação de produtos orgânicos visa conquistar maior credibilidade dos consumidores e conferir maior transparência às práticas e aos princípios utilizados na produção orgânica. A certificação é outorgada por diferentes instituições no País, as quais possuem normas específicas para a concessão do seu selo de garantia. O organismo certificador tem a função de desenhar um método que seja capaz de minimizar o risco de fraude e que seja responsável, perante a lei, pelo cumprimento rigoroso do método apresentado a seu credenciador.

Diversos são os desafios inerentes aos elos da cadeia produtiva de orgânicos, principalmente os relacionados à comercialização desse tipo de produto, sobretudo a respeito da certificação. Ainda que existam alguns mecanismos de avaliação que conferem conformidade orgânica à produção, o índice de propriedades certificadas no Brasil ainda é baixo (IBGE, 2006).

De acordo com Komori et al. (2007), analisando a agricultura orgânica no estado de Mato Grosso do Sul, existem muitas dificuldades na comercialização dos alimentos orgânicos prove- 
nientes das pequenas propriedades familiares. Muitos produtores acabam excluídos do processo, devido à necessidade de certificação, pois é inviável financeiramente para muitos agricultores.

Foram vários os motivos que levaram os produtores orgânicos da Agroufam a iniciarem esse modo de produção, destacando-se como principais: fidelidade dos consumidores; grande quantidade de agrotóxicos utilizada por ano; grande procura por produtos de origem agroecológica; a produção orgânica é mais saudável; doenças que podem ser causadas por uma alimentação inadequada.

Segundo os produtores, os consumidores são fiéis, e, no período de comercialização, existe grande demanda por produtos de origem agroecológica. As razões que levam as pessoas a comprarem produtos orgânicos são inúmeras, segundo constatação teórica. $O$ fundamental para esse público são os nutrientes e alimentos livres de agrotóxicos, que beneficiam a saúde e preservam a natureza. Além da preocupação com o desenvolvimento sustentável e a inclusão social, esses consumidores são fiéis em suas escolhas por acreditarem que os alimentos orgânicos são capazes de conduzi-los a valores como bem-estar, prazer e qualidade de vida, possibilitando, assim, viver mais e melhor (GONÇALVES et al., 2016).

Um dos agricultores afirmou que sua principal motivação foi a grande quantidade de agrotóxicos que é utilizada por ano. Segundo dados do IBGE (2015), entre 2002 e 2012, a comercialização de agrotóxicos e produtos afins aumentou de $2,7 \mathrm{~kg} / \mathrm{ha}$ para $6,9 \mathrm{~kg} / \mathrm{ha}$, um aumento de $155 \%$. Grande parte dos produtos, cerca de $65 \%$, foram classificados como perigosos, quase $30 \%$ como muito perigoso e apenas $1 \%$ dos produtos como pouco perigosos ao meio ambiente.

Segundo a Organização Mundial da Saúde (OMS), entre os países em desenvolvimento, os agrotóxicos causam anualmente 70 mil intoxicações agudas e crônicas (ROSSI, 2015). O volume 113 dos estudos da IARC/WHO foi realizado por um time de excelência composto por 26 pesquisadores de 13 diferentes países e foi publicado na revista The Lancet Oncology, no dia 22 de junho de 2015 (DIAS, 2015).

\section{Conclusão}

Nas entrevistas foi possível observar que o principal problema é a ausência de auxílio técnico, seja este na área de fitotecnia ou na própria gestão do negócio. Apesar de os agricultores estarem mais conscientes dos riscos dos agrotóxicos à saúde e vendo a oportunidade de adentrar em um nicho de mercado que possui consumidores fiéis, a falta de assistência vem dificultando a expansão e adoção do sistema de produção orgânico.

Outros grandes gargalos apontados são os custos elevados da certificação e a logística da produção, que desmotivam a permanência no sistema, pois são repassados diretamente ao consumidor final, causando baixo consumo desse tipo de produto pela população com menor poder aquisitivo. Apesar das dificuldades, o produtor vem se mostrando otimista e mais consciente, reforçando o papel da feira Agroufam na produção orgânica do estado. 


\section{Referências}

BARBOSA, W. F.; SOUSA, E. P. Agricultura orgânica no Brasil: características e desafios. Revista Economia \& Tecnologia (RET), v. 8, n. 4, p. 67-74, 2012.

BARROS, J. D. S.; SILVA, M. F. P. Práticas agrícolas sustentáveis como alternativas ao modelo hegemônico de produção agrícola. Sociedade e Desenvolvimento Rural, v. 4, n. 2, p. 89-103, set. 2010.

CAMPANHOLA, C.; VALARINI, P. J. A agricultura orgânica e seu potencial para o pequeno produtor. Cadernos de Ciência \& Tecnologia, v. 18, n. 3, p. 69-110, 2001.

DIAS, B. OMS divulga nova classificação de venenos relacionados ao câncer. Abrasco, 24 jun. 2015. Disponível em: https://www.abrasco.org.br/site/outras-noticias/saude-da-populacao/oms-divulga-nova-classificacao-de-pesticidas-relacionados-ao-cancer/11545/. Acesso em: 15 maio 2019.

FINATTO, R. A.; CORRÊA, W. K. Desafios e perspectivas para a comercialização de produtos de base agroecológica: o caso do município de Pelotas/RS. Revista Brasileira de Agroecologia, v. 5, n. 1, p. 95 $105,2010$.

FRANÇA, A. R. M. et. al. Economia solidária e desenvolvimento rural: a agricultura familiar e sua diversificação na Rede Xique-Xique (RN). In: SOCIEDADE BRASILEIRA DE ECONOMIA, ADMINISTRAÇÃO E SOCIOLOGIA RURAL-SOBER NORDESTE, 2010, Crato, CE. Anais... Crato: SOBER, 2010. p. 1-15.

FREITAS, J. C. Agricultura sustentável: uma análise comparativa dos fatores de produção entre agricultura orgânica e agricultura convencional. 2002. Dissertação (Mestrado em Economia) - Departamento de Economia, Universidade de Brasília, Brasília, DF.

FREITAS, J. de; ANDRADE, L.; BORDEAUX-REGO, R. Crescimento orgânico: uma análise da estratégia de expansão das lojas Americanas. In: CONGRESSO NACIONAL DE EXCELÊNCIA EM GESTÃO, 11., 2015, Rio de Janeiro. Anais... The Brazilian Journal of Operations \& Production Management, v. 11, p. 1-15, 2015.

GOMES, J. B. P.; PADOVAN, M. P.; PADOVAN, D. S. S. Produção orgânica no âmbito da agricultura familiar em Mato Grosso do Sul. Revista do Desenvolvimento Rural (Redes), v. 22, n. 3, 2017.

GONÇALVES, F. O.; ROLIM, M. F. S.; ROSA, T. M. Motivações para o consumo de alimentos orgânicos - possibilidades do Distrito Federal. Brasília, DF: CODEPLAN - Políticas de Promoção Social, 2016.

IBGE. Censo Agropecuário 2006. Rio de Janeiro, 2006. 777 p. Disponível em: https://biblioteca.ibge. gov.br/visualizacao/periodicos/51/agro_2006.pdf. Acesso em: 20 jun. 2018.

IBGE. Indicadores de desenvolvimento sustentável. Rio de Janeiro, 2015. 352 p. Disponível em: https:// biblioteca.ibge.gov.br/visualizacao/livros/liv94254.pdf . Acesso em: 25 abr. 2019.

GOMES, J. B. P.; PADOVAN, M. P.; PADOVAN, D. S. S. Produção orgânica no âmbito da agricultura familiar em Mato Grosso do Sul. Revista do Desenvolvimento Rural (Redes), v. 22, n. 3, 2017.

KOMORI, O. M. et al. Núcleo de Agroecologia de Mato Grosso do Sul. Revista Brasileira de Agroecologia, v. 2, n. 1, p. 1746-1749, 2007.

LOMBA, R. M.; SILVA, I. C.; FILOCREAO, A. S. M. Assistência técnica e extensão rural na agricultura familiar do Estado do Amapá, Brasil. In: ENCONTRO DE GEÓGRAFOS DA AMÉRICA LATINA, 14., 2013, Lima. Articulos e ponencias. Lima: UGI, 2013. 
MAZZOLENI, E. M.; NOGUEIRA, J. M. Agricultura orgânica: características básicas do seu produtor. Revista de Economia e Sociologia Rural, v. 44, n. 2, Apr./June. 2006.

PENTEADO, S. R. Introdução à agricultura orgânica: normas e técnicas de cultivo. Campinas: Editora Grafimagem, 2000. 110 p.

ROSSI, M. O “alarmante” uso de agrotóxicos no Brasil atinge 70\% dos alimentos. EI País, 30 abr. 2015. SANTOS, G. C.; MONTEIRO, G. Sistema orgânico de produção de alimentos. Alimento \& Nutrição, v. 15, n. 1, p. 73-86, 2004. 
